\title{
Observation of the optical spin Hall effect
}

\author{
C. LEYDER ${ }^{1 *}$, M. ROMANELLI', J. PH. KARR' ${ }^{1}$ E. GIACOBINO'1, T. C. H. LIEW², M. M. GLAZOV ${ }^{3}$, \\ A. V. KAVOKIN², G. MALPUECH ${ }^{4}$ AND A. BRAMATI ${ }^{1 *}$ \\ ${ }^{1}$ Laboratoire Kastler Brossel, Université Paris 6, Ecole Normale Supérieure et CNRS, UPMC Case 74, 4 place Jussieu, 75252 Paris Cedex 05, France \\ ${ }^{2}$ Physics and Astronomy School, University of Southampton, Highfield, Southampton, S017 1BJ, UK \\ ${ }^{3}$ A.F. Ioffe Physico-Technical Institute, Russian Academy of Sciences, 194021 St-Petersburg, Russia \\ ${ }^{4}$ LASMEA, UMR6602 CNRS/Université Blaise Pascal, 24 Avenue des Landais, 63177 Aubière, France \\ *e-mail: charles.leyder@spectro.jussieu.fr; bramati@spectro.jussieu.fr
}

The spin Hall effect consists of the generation of a spin current perpendicular to the charge current flow. Thirty-five years after its prediction by Dyakonov and Perel', it is the focus of experimental and theoretical investigations and constitutes one of the most remarkable effects of spintronics. Owing to scattering and dephasing in electronic gases, it is difficult to observe and has only been demonstrated for the first time a few years ago ${ }^{2-5}$. Recently, three of us have predicted the optical spin Hall effect ${ }^{6}$, which consists of a separation in real space and momentum space of spin-polarized exciton-polaritons generated by a laser in a semiconductor microcavity ${ }^{7}$. The separation takes place owing to a combination of elastic scattering of exciton-polaritons by structural disorder and an effective magnetic field coming from polarization splitting of the polariton states. The excitonic spin current is controlled by the linear polarization of the laser pump. Here, we report the first experimental evidence for this effect and demonstrate propagation of polariton spin currents over $100 \mu \mathrm{m}$ in a high-quality GaAs/AlGaAs quantum microcavity. By rotating the polarization plane of the exciting light, we were able to switch the directions of the spin currents.

The spin carries a quantum bit of information, which makes spin-based devices, also called spintronic devices, extremely promising for quantum-information processing ${ }^{8}$. More specifically, the spin Hall effect (SHE) has recently raised an increasing interest owing to its potentialities in spintronics ${ }^{2,3}$. In a similar way to the Hall effect, in which a magnetic field causes a particle to deviate depending on its charge, the spin Hall effect results in a spin current owing to the spin-dependent scattering of electrons by charged impurities or other defects (extrinsic $\mathrm{SHE}^{1,9}$ ) or to the spin-orbit effects on the carrier energy dispersion (intrinsic $\left.\mathrm{SHE}^{10,11}\right)$. It leads to a separation in real space and momentum space between spin-up and spin-down electrons and thus enables control of electron spin in semiconductor spintronic devices ${ }^{12}$. Optical $^{2,3}$ and electronic ${ }^{4}$ measurements of the extrinsic SHE have been reported. Although successfully generated, electronic spin currents are subject to rapid dephasing and decay, mostly due to electron scattering. Recent theoretical studies ${ }^{5,13}$ show that the intrinsic SHE is fully suppressed in macroscopic-sized samples because of electron scattering, which directs the system towards equilibrium. The use of neutral particles as carriers for spin currents is an alternative approach that may overcome the problem of dephasing. Here, we demonstrate the spin currents carried by (neutral) exciton-polaritons in a semiconductor microcavity and propagating coherently over $100 \mu \mathrm{m}$. We demonstrate for the first time experimentally that the spin currents can be controlled by an optical spin Hall effect (OSHE), which exhibits remarkable analogy to the spin Hall effect.

Exciton-polaritons (or polaritons for short) are the 'mixed' exciton-photon quasiparticles associated with the normal modes of semiconductor microcavities. They have been studied extensively since the discovery of strong light-matter coupling in these systems in 1992 (ref. 14). Many unique properties of polaritons are derived from their non-parabolic in-plane dispersion and composite bosonic nature ${ }^{7}$. Polaritons are particularly suitable as spin current carriers owing to their long coherence length, of the order of $100 \mu \mathrm{m}$ (ref. 15), compared with that of electronic gases. Furthermore, the recent observation of Bose-Einstein condensation of polaritons in microcavities ${ }^{16}$ confirms their high potential for fascinating quantum coherent effects ${ }^{17,18}$.

Polaritons can be created by directly shining a laser onto the microcavity where the angle of incidence determines their in-plane wavevector. The polaritons scatter elastically with disorder in the system and redistribute around an elastic circle in reciprocal space (the modulus of the in-plane wavevector is conserved). A splitting in energy between polaritons with transverse electric and transverse magnetic linear polarizations causes different spin currents to develop in different quadrants of the elastic circle. This OSHE can be considered as the result of an effective magnetic field acting on a pseudospin.

The polariton spin (angular momentum) has \pm 1 projections on the growth axis of the microcavity. Therefore, the polariton can be treated as a two-level system with an effective pseudospin $S=1 / 2$. Furthermore, there is a strict correspondence between the polariton pseudospin vector in the Poincaré sphere (Fig. 1a) and the polarization of light emitted by the microcavity at a given angle. Similar to an angular momentum, the polariton spin state can be controlled by an appropriate polarized excitation and can be analysed by a polarization-resolved detection. The polarization of light can be fully characterized by measuring a set of values, the so-called Stokes parameters ${ }^{19}$ that correspond to the following polarization degrees:

$$
\rho_{\mathrm{l}}=\frac{I_{\leftrightarrow}-I_{\uparrow}}{I_{\leftrightarrow}+I_{\uparrow}}, \quad \rho_{\mathrm{d}}=\frac{I_{+45^{\circ}}-I_{-45^{\circ}}}{I_{+45^{\circ}}+I_{-45^{\circ}}}, \quad \rho_{\mathrm{c}}=\frac{I_{\sigma^{+}}-I_{\sigma^{-}}}{I_{\sigma^{+}}+I_{\sigma^{-}}} .
$$

where 1 and $\mathrm{d}$ denote the linear polarization degree measured in the $x y$ and diagonal axes, respectively, and $c$ denotes the circular polarization. $I_{\leftrightarrow}, I_{\uparrow}, I_{+45^{\circ}}$ and $I_{-45^{\circ}}$ represent the intensities 

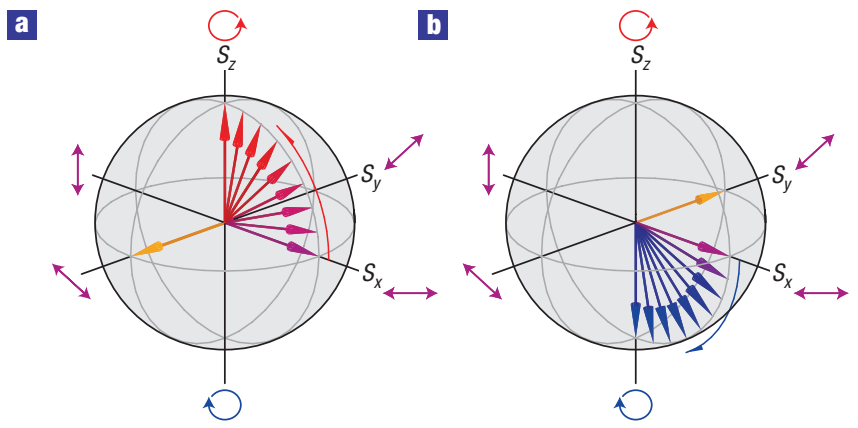

G

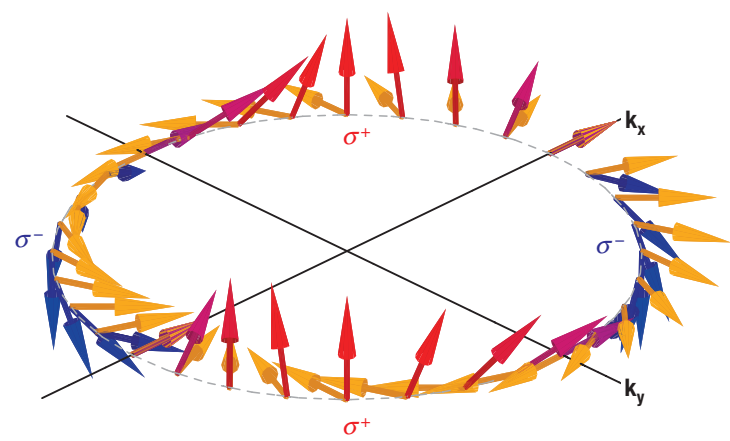

Figure 1 Angle-dependent evolution of the pseudospin vectors. a, The pseudospin vector represents the polariton polarization and lies on the surface of the Poincaré sphere for a pure polarized state. Initially the pseudospin lies in the $x$-direction (purple arrow), representing a horizontal linearly polarized state. If the effective magnetic field representing the longitudinal-transverse splitting lies in the $y$ direction (orange arrow) then the pseudospin rotates until it is parallel to the $z$ axis (red arrow); the initial linear polarization becomes a $\sigma^{+}$polarization. $\mathbf{b}$, If the effective magnetic field lies in the opposite direction, then the pseudospin is rotated until it is antiparallel to the $z$ axis (blue arrow) and the state becomes $\sigma^{-}$polarized. c, Although the effective magnetic field always lies in the microcavity plane, its direction is dependent on the angle of the wavevector (orange arrows). The evolution of pseudospins is therefore anisotropic. For an initial linearly polarized state of polaritons around the elastic circle, the pseudospins will all lie along the $x$ axis (not shown). After some time, all of the pseudospins point in different directions corresponding to different polarizations. Red arrows represent $\sigma^{+}$polarizations; blue arrows represent $\sigma^{-}$polarizations; purple arrows represent linear polarizations.

measured through linear polarizers oriented at $0^{\circ}, 90^{\circ}, 45^{\circ}$ and $-45^{\circ}$ to the $x$ axis; $I_{\sigma^{+}}$and $I_{\sigma^{-}}$represent the intensities measured in the circular polarization basis. The OSHE is the precession of the pseudospin of the polariton around an effective magnetic field, $\boldsymbol{\Omega}$, owing to the longitudinal-transverse splitting, $\Delta_{\mathrm{LT}}$ (ref. 20), which is induced by the long-range part of the exciton exchange interaction $^{21}$ and by the transverse electric-transverse magnetic splitting of the photon mode of the cavity ${ }^{22}$. The value of this magnetic field is proportional to $\Delta_{\text {LT }}$ and its direction depends on the polar angle, $\theta$, of the polariton in-plane wavevector, $\mathbf{k}=\left(\mathbf{k}_{\mathbf{x}}, \mathbf{k}_{\mathbf{y}}\right)$ in momentum space $^{6}$ :

$$
\Omega_{x}=\frac{\Delta_{\mathrm{LT}}(k)}{\hbar} \cos 2 \theta, \quad \Omega_{y}=\frac{\Delta_{\mathrm{LT}}(k)}{\hbar} \sin 2 \theta .
$$

The evolution of a pseudospin is critically dependent on the orientation of $\boldsymbol{\Omega}$. Figure 1a,b shows the evolution of a pseudospin having an initial linear polarization into a left $\left(\sigma^{+}\right)$or right $\left(\sigma^{-}\right)$ circular polarization depending on the orientation of $\boldsymbol{\Omega}$. Figure 1c shows the dependence of the $\boldsymbol{\Omega}$ orientation on the orientation of

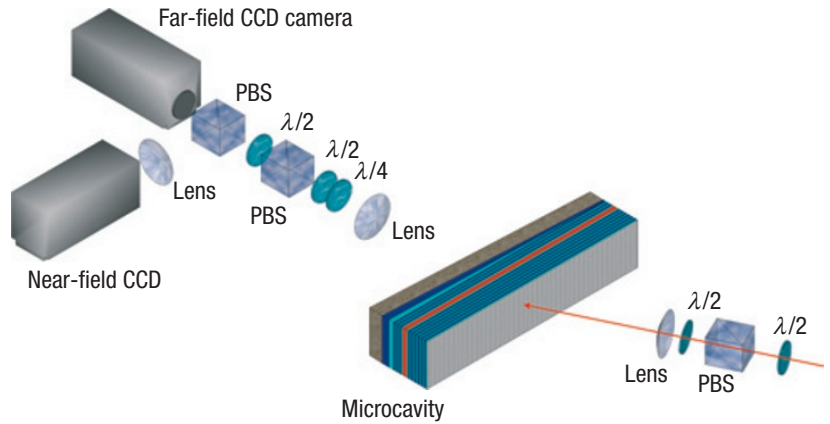

Figure 2 Experimental set-up. A linearly polarized pump beam, $\mathbf{k}_{\mathrm{p}}$, with an incidence angle of $12^{\circ}$ is focused on the microcavity sample. The microcavity emission is collimated by a lens $(f=50 \mathrm{~mm})$. The emission is then resolved in polarization and split into two parts. One is imaged on a CCD camera to obtain the far-field. The other is sent through a lens to another CCD camera to obtain the near-field. PBS: Polarizing beam splitter.

$\mathbf{k}$ and its consequences on the pseudospin evolution. Similar to the role of the Rashba field in the intrinsic $\mathrm{SHE}^{10,11}$, the effective magnetic field causes different polarizations to develop in different directions in the OSHE. Recently, the existence of this field has been demonstrated in experiments on linear polarization conversion in the ballistic transport of exciton-polaritons ${ }^{23,24}$.

The evolution of the pseudospin can be well described by the following precession equation:

$$
\frac{\partial \mathbf{S}}{\partial t}=\mathbf{S} \wedge \boldsymbol{\Omega}(k)+\frac{\mathbf{S}_{0}}{\tau_{1}}-\frac{\mathbf{S}}{\tau} .
$$

The first term describes the precession of the pseudospin. The second term describes the input term due to the Rayleigh scattering from the initial state $\left(\tau_{1}\right.$ is the Rayleigh scattering time constant). The last term describes the radiative relaxation ( $\tau$ is the polariton lifetime).

Resonant Rayleigh scattering is due to the static disorder in the microcavity ${ }^{25}$, which is inevitably present in any realistic microcavity owing to thickness variations in the cavity and Bragg mirror layers. Thus, when an initial polariton state with a given in-plane wavevector, $\mathbf{k}=\left(\mathbf{k}_{\mathbf{x}}, \mathbf{k}_{\mathbf{y}}\right)$, and a given polarization state, transverse magnetic or transverse electric, is created, it is efficiently scattered towards states with in-plane wavevectors different from the initial one and with the same polarization state. As the modulus of $\mathbf{k}$ is conserved, this scattering gives rise to the Rayleigh scattering ring in the far-field emission ${ }^{26,27}$. Then the anisotropy in the orientation of the effective magnetic field creates an anisotropy in the polarization: in two opposite quadrants of the elastic circle, $\sigma^{+}$ polarization appears, whereas in the two others, $\sigma^{-}$polarization appears (see Fig. 1c). The spin separation on the elastic circle leads to spin separation in real space owing to ballistic propagation of the polaritons, thus the polariton spin currents are formed.

To detect the OSHE, we use the experimental set-up shown schematically in Fig. 2. We use a continuous titanium-sapphire pump laser at $836 \mathrm{~nm}$ with an angle of incidence of $12^{\circ}$. The polarization of the pump is linear and can be adjusted to transverse magnetic or transverse electric polarization. The far-field emission transmitted by the microcavity is polarization resolved and imaged on a $1,024 \times 1,024 \mathrm{CCD}$ (charge-coupled device) camera. This set-up allows a precise measurement of the Stokes parameters of the light in the far-field, thus providing information on the pseudospin of a polariton state with a given in-plane wavevector. 

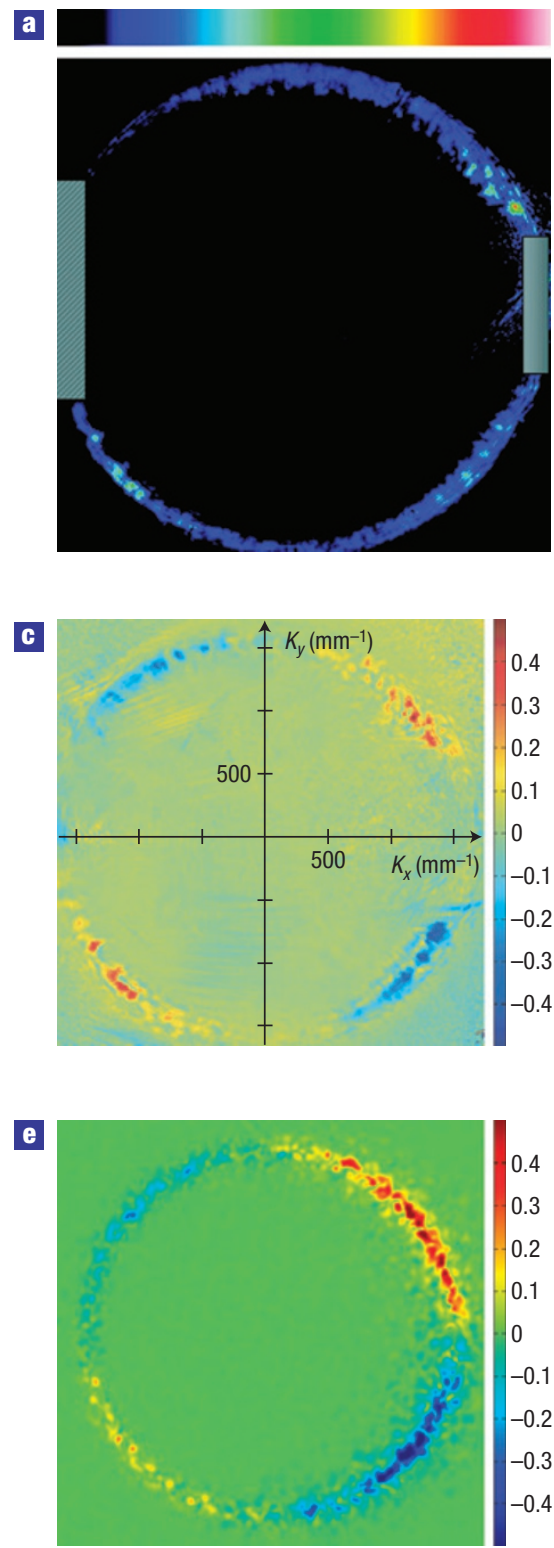
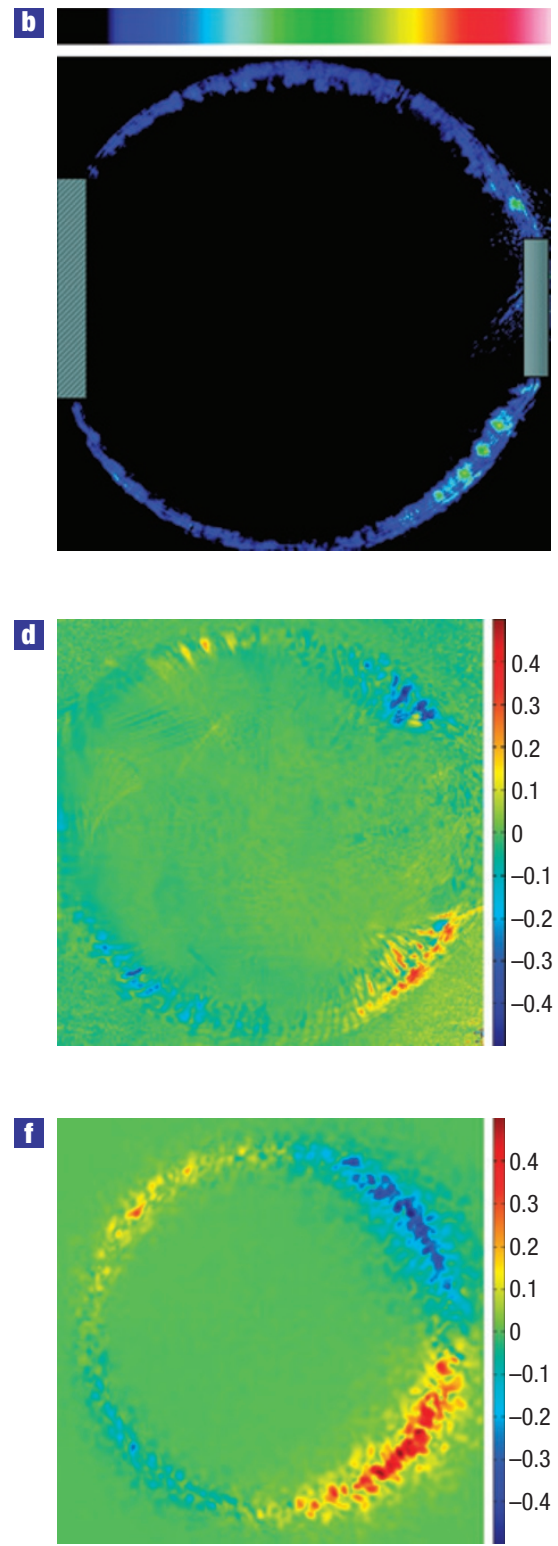

Figure 3 Observation of the OSHE in reciprocal space and comparison with theory. a, The $\sigma^{+}$component of the emission from the microcavity in the far-field. The intensity scale is linear. The transverse magnetic $(x)$ polarized pump beam (on the right) and the backscattering (on the left) are blocked to protect the CCD from over exposure. The polaritons undergo resonant Rayleigh scattering and become distributed around an elastic circle. The speckles are a characteristic of Rayleigh scattering in microcavities ${ }^{24} . \mathbf{b}$, The complementary $\sigma^{-}$polarized far-field emission. c. The measured degree of circular polarization, $\rho_{\mathrm{c}}$, in the far-field emission for a transverse magnetic $(x)$ polarized pump. The colour scale is linear, distinguishing $\sigma^{+}$polarizations in red and $\sigma^{-}$polarizations in blue. The anisotropy in the polarized emission is in agreement with Fig. 1. d, The same as in $\mathbf{c}$ but for a transverse electric $(y)$ polarized pump. Note that the regions of $\sigma^{+}$and $\sigma^{-}$polarizations are interchanged. e, Prediction of the far-field polarization degree from numerical solution of the two-component Schrödinger equation with a random potential for the case of a transverse magnetic $(x)$ polarized pump. The polarization degrees vary in an identical way around the elastic circle to that measured in $\mathbf{c}$. f The same as in e but for a transverse electric $(y)$ polarized pump. Again there is an excellent agreement with the experimental result (d). In our calculation, we used the following parameters: $k_{\mathrm{p}}=1,560 \mathrm{~mm}^{-1}, \Delta_{\mathrm{LT}}\left(k_{\mathrm{p}}\right)=0.05 \mathrm{meV}$, $\tau=10 \mathrm{ps}$, cavity photon effective mass, $m_{\mathrm{C}}=10^{-5} m_{\mathrm{e}}$ ( $m_{\mathrm{e}}$ is the free electron mass), exciton effective mass, $m_{\mathrm{exc}}=0.22 m_{\mathrm{e}}$, cavity-exciton detuning, $\delta=0.3$ meV, Rabi splitting $=5.1 \mathrm{meV}$ and pump spot diameter $50 \mu \mathrm{m}$. The disorder potential was generated using the procedure in ref. 28 to generate a single-layer disorder potential. The potential had an r.m.s. amplitude of $0.5 \mathrm{meV}$ and an isotropic correlation length of $0.75 \mu \mathrm{m}$.

The polariton spin currents in real space have been detected using a near-field polarization-resolved set-up. A complete description of the experiment is available in the Supplementary Information.

The microcavity was excited by a transverse magnetic polarized pump resonant with the lower polariton branch with a slight cavity-exciton detuning of $0.3 \mathrm{meV}$. The intensities $I_{\sigma+}$ and $I_{\sigma-}$ of the light transmitted by the microcavity were imaged and measured successively using a CCD camera (Fig. 3a,b). The value of the circular Stokes parameter, $\rho_{\mathrm{c}}$, was calculated for each pixel using equation (1). The result is shown in Fig. 3c. The dependence on $\theta$ of the circular parameter is in excellent agreement with the theoretical predictions shown in Fig. 1c. The maxima are located 

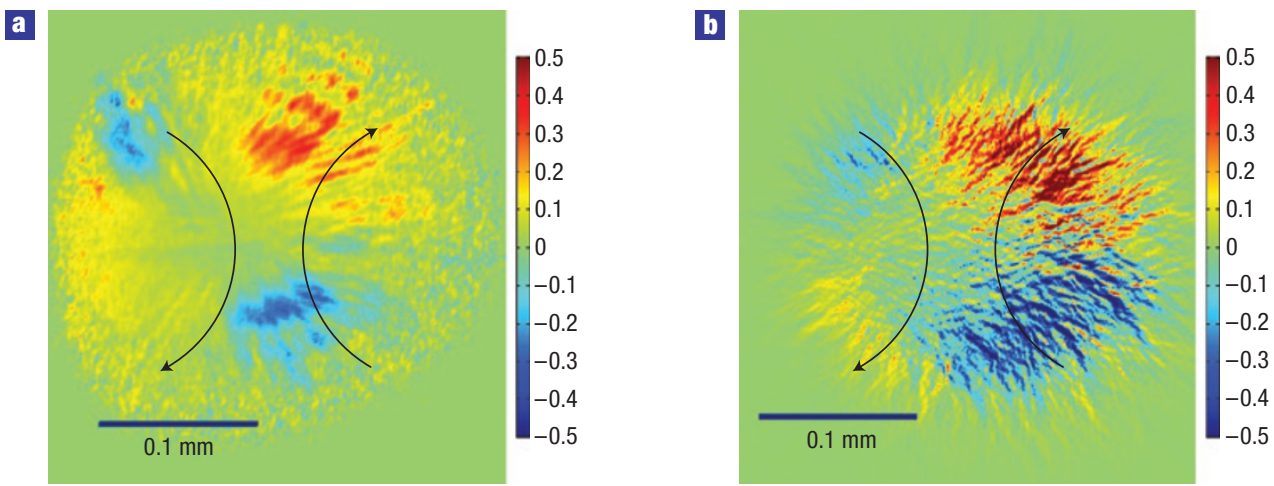

Figure 4 Polariton spin currents in the real space. a, The circular polarization degree of the spatially resolved secondary emission by the microcavity for the case of a transverse magnetic polarized pump. b. Results of simulation obtained by a numerical Fourier transform of the polariton distributions in the reciprocal space (Fig. 3). Near the centre of the pump spot, any circular polarization of polaritons is overwhelmed by the linear polarization of the pump. However, once polaritons have migrated from the centre of the pump spot, their polarization depends on the orientation of their wavevector; the OSHE causes the different spin polarizations to separate in real space on the edges of the pump spot. The directions of the $\sigma^{+}$and $\sigma^{-}$spin currents are shown by black arrows.

at $\pm \pi / 4$ and $\pm 3 \pi / 4$ and the sign of $\rho_{\mathrm{c}}$ is reversed between $\pi / 4$ and $3 \pi / 4$. For our experimental values of $\Delta_{\mathrm{LT}}(0.05 \mathrm{meV})$ and $\tau$ ( $\sim 10 \mathrm{ps}$ ), the maximal expected value of $\rho_{\mathrm{c}}$ is then close to 0.45 in excellent agreement with our experimental value of $0.48 \pm 0.03$. Similar results are shown in Fig. 3d, where the experiment is carried out in the same conditions but with a transverse electric polarized excitation. The sign of $\rho_{c}$ is then clearly reversed. We have also checked that $\rho_{\mathrm{c}}$ is very close to zero on the whole farfield if another spot with $\Delta_{\mathrm{LT}}=0$ of the microcavity sample is excited. These experimental results are also in very good agreement with numerical simulations based on the solution of a twocomponent Schrödinger equation for the exciton polaritons that accounts for the disorder potential responsible for the Rayleigh scattering (Fig. 3e,f). The details of our formalism are given in the Supplementary Information.

Figure $4 \mathrm{a}$ shows the near-field images of the circular polarization degree of emission by the microcavity excited by transverse magnetic polarized light. It can be seen that the polaritons carrying the $\sigma^{+}$or $\sigma^{-}$polarization propagate ballistically over about $100 \mu \mathrm{m}$, which means that macroscopic spin currents are generated. Their direction is controlled by the polarization of excitation light. This observation is in excellent agreement with the results of the simulation (Fig. 4b).

In the OSHE, the spin currents are carried by electrically neutral exciton-polaritons, which are subject to bosonic statistics. This represents two important advantages with respect to the electronic SHE: there is no dephasing owing to the Coulomb repulsion of the carriers and there is a possibility to create superfluid spin currents. Clearly, our microcavity acts as an optically controlled spin generator allowing for integration into photonic circuits. Future research is needed to investigate the OSHE in the nonlinear regime and superfluid spin currents.

\section{Received 3 May 2007; accepted 14 June 2007; published 15 July 2007.}

\section{References}

1. Dyakonov, M. I. \& Perel, V. I. Current induced spin orientation of electrons in semiconductors. Phys. Lett. A 35, 459-460 (1971)

2. Kato, Y. K., Myers, R. C., Gossard, A. C. \& Awshalom, D. D. Observation of the spin Hall effect in semiconductors. Science 306, 1910-1913 (2004).

3. Wunderlich, J., Kaestner, B., Sinova, J. \& Jungwirth, T. Experimental observation of the spin-Hall effect in a two-dimensional spin-orbit coupled semiconductor system. Phys. Rev. Lett. 94, 047204 (2005).

4. Valenzuela, S. O. \& Tinkham, M. Direct electronic measurement of the spin Hall effect. Nature $\mathbf{4 4 2}$ $176-179(2006)$
5. Mishchenko, E. G., Shytov, A. V. \& Halperin, B. I. Spin current and polarization in impure two-dimensional electron systems with spin-orbit coupling. Phys. Rev. Lett. 93, 226602 (2004).

6. Kavokin, A. V., Malpuech, G. \& Glazov, M. M. Optical spin Hall effect. Phys. Rev. Lett. 95, 136601 (2005).

7. Kavokin, A. V. \& Malpuech, G. Cavity Polaritons (Elsevier, Amsterdam, 2003).

8. Awshalom, D. D., Loss, D. \& Samarth, N. Semiconductor Spintronics and Quantum Computation (Springer, Berlin, 2002)

9. Hirsch, J. E. Spin Hall effect. Phys. Rev. Lett. 83, 1834-1837 (1999).

10. Murakami, S., Nagaosa, N. \& Zhang, S.-C. Dissipationless quantum spin current at room temperature. Science 301, 1348-1351 (2003).

11. Sinova, J. et al. Universal intrinsic spin Hall effect. Phys. Rev. Lett. 92, 126603-126606 (2004).

12. Sih, V. et al. Spatial imaging of the spin Hall effect and current-induced polarization in two-dimensional electron gases. Nature Phys. 1, 31-35 (2005).

13. Chalaev, O. \& Loss, D. Spin-Hall conductivity due to Rashba spin-orbit interaction in disordered systems. Phys. Rev. B 71, 245318-245325 (2005).

14. Weisbuch, C., Nishioka, M., Ishikawa, A. \& Arakawa, Y. Observation of the coupled exciton-photon mode splitting in a semiconductor quantum microcavity. Phys. Rev. Lett. 69, 3314-3317 (1992).

15. Freixanet, T., Sermage, B., Tiberj, A. \& Planel, R. In-plane propagation of excitonic cavity polaritons. Phys. Rev. B 61, 7233-7236 (2000).

16. Kasprzak, J. et al. Bose-Einstein condensation of exciton-polaritons. Nature 443, 409-414 (2006).

17. Karr, J. Ph., Baas, A., Houdré, H. \& Giacobino, E. Squeezing in semiconductor microcavities in the strong coupling regime. Phys. Rev. A 69, 031802(R) (2004).

18. Baas, A., Karr, J. Ph., Romanelli, M., Bramati, A. \& Giacobino, E. Quantum degeneracy of microcavity polaritons. Phys. Rev. Lett. 96, 176401 (2006)

19. Born, M. \& Wolf, E. Principles of Optics (Cambridge Univ. Press, Cambridge, 1999).

20. Shelykh, I., Malpuech, G., Kavokin, K. V., Kavokin, A. V. \& Bigenwald, P. Spin dynamics of exciton polaritons in microcavities. Phys. Rev. B 70, 115301 (2004).

21. Maialle, Z. M., de Andrada e Silva, E. A. \& Sham, L. J. Exciton spin dynamics in quantum wells. Phys Rev. B 47, 15776 (1993).

22. Panzarini, G. et al. Exciton-light coupling in single and coupled semiconductor microcavities: Polariton dispersion and polarization splitting. Phys. Rev. B 59, 5082-5089 (1999).

23. Langbein, W. Proc. 26th Int. Conf. on Physics of Semiconductors 112 (Institute of Physics, Bristol, 2003)

24. Langbein, W. et al. Polarization beats in ballistic propagation of exciton-polaritons in microcavities. Phys. Rev. B 75, 075323-075331 (2007).

25. Haacke, S. Resonant Rayleigh scattering by Wannier excitons in a two-dimensional disordered potential. Rep. Prog. Phys. 64, 737-776 (2001).

26. Langbein, W. \& Hvam, J. M. Elastic dynamics of cavity polaritons. Phys. Rev. Lett. 88, 047401-047404 (2002).

27. Houdré, R., Weisbuch, C., Stanley, R. P., Oesterle, U. \& Ilegems, M. Coherence effects in light scattering of two-dimensional photonic disordered systems: Elastic scattering of cavity polaritons. Phys. Rev. B 61, R13333-R13336 (2000)

28. Savona, V. \& Langbein, W. Realistic heterointerface model for excitonic states in growth-interrupted GaAs quantum wells. Phys. Rev. B 74, 75311 (2006).

Acknowledgements

T.C.H.L. was supported by E.P.S.R.C. A.V.K. acknowledges support from the EU project STIMSCAT (contract 517769). We are very grateful to R. Houdré (Ecole Polytechnique Fédérale de Lausanne, Switzerland) for providing the high-quality microcavity sample.

Correspondence and requests for materials should be addressed to C.L. or A.B.

Supplementary Information accompanies this paper on www.nature.com/naturephysics.

\section{Author contributions}

C.L., M.R., J.P.K., E.G. and A.B. contributed to the experimental work, A.V.K. developed the theoretical concept and T.C.H.L., M.M.G. and G.M. contributed to the theoretical work.

Competing financial interests

The authors declare no competing financial interests.

Reprints and permission information is available online at http://npg.nature.com/reprintsandpermissions/ 